\title{
Research Challenges in Parallel and Distributed Simulation
}

\author{
RICHARD M. FUJIMOTO, Georgia Institute of Technology
}

\begin{abstract}
The parallel and distributed simulation field has evolved and grown from its origins in the 1970s and 1980s and remains an active field of research to this day. A brief overview of research in the field is presented. Future research topics are explored including areas such as problem-driven simulation of large-scale systems and complex networks, exploitation of graphical processing unit hardware and cloud computing environments, predictive online simulation for system management and optimization, power and energy consumption in mobile platforms and data centers, and composition of heterogeneous simulations.
\end{abstract}

CCS Concepts: • Computing methodologies $\rightarrow$ Discrete-event simulation; Distributed simulation; Massively parallel and high-performance simulations

Additional Key Words and Phrases: Research challenges, parallel discrete event simulation

ACM Reference Format:

Richard M. Fujimoto. 2016. Research challenges in parallel and distributed simulation. ACM Trans. Model. Comput. Simul. 26, 4, Article 22 (May 2016), 29 pages.

DOI: http://dx.doi.org/10.1145/2866577

\section{INTRODUCTION AND HISTORICAL PERSPECTIVE}

The parallel and distributed simulation field emerged in the 1970s and 1980s from two distinct research communities. On the one hand, the parallel discrete event simulation (PDES) community was concerned with accelerating the execution of discrete event simulations through the exploitation of high-performance computing platforms. In approximately the same time frame, the distributed simulation community formed, growing out of research and development efforts in the defense community that focused on interconnecting simulations executing on computers interconnected via local and wide area networks. While there are important differences between work in PDES and distributed simulation, there are also many common issues and problems. Here, we informally define parallel and distributed simulation as a field focusing on the execution of a simulation program on multiple processors in platforms ranging from tightly coupled parallel computers to loosely coupled computers connected via a network. The particular emphasis here is on discrete event simulations used for the analysis of systems.

\subsection{Parallel Discrete Event Simulation}

PDES is concerned with distributing a single execution of a discrete event simulation program across multiple processors in a high-performance computing system. Such platforms include shared-memory multiprocessors and message-based cluster computers. The central goal of PDES is typically to accelerate the execution of the simulation.

Author's address: R. M. Fujimoto, School of Computational Science and Engineering, Georgia Institute of Technology, Atlanta, Georgia 30332-0280.

Permission to make digital or hard copies of part or all of this work for personal or classroom use is granted without fee provided that copies are not made or distributed for profit or commercial advantage and that copies show this notice on the first page or initial screen of a display along with the full citation. Copyrights for components of this work owned by others than ACM must be honored. Abstracting with credit is permitted. To copy otherwise, to republish, to post on servers, to redistribute to lists, or to use any component of this work in other works requires prior specific permission and/or a fee. Permissions may be requested from Publications Dept., ACM, Inc., 2 Penn Plaza, Suite 701, New York, NY 10121-0701 USA, fax +1 (212) 869-0481, or permissions@acm.org.

(c) 2016 ACM 1049-3301/2016/05-ART22 $\$ 15.00$

DOI: http://dx.doi.org/10.1145/2866577 
A discrete event simulation captures the behavior of an actual or envisioned system over time. Unlike other simulations that operate in a time-stepped fashion and evolve the state of the system from one time step to the next, in a discrete event simulation, changes in system state occur at distinct, typically irregular, points in simulation time. A sequential discrete event simulation program includes two fundamental concepts: state variables that capture the state of the system being modeled, and event computations or simply events that transition the state variables from one state to the next. In a discrete event simulation, changes to simulation state occur only through event computations. Each event contains a timestamp that represents a point in simulation time at which the state transition occurs. For example, a simulation of an airport might include state variables representing the status of the runway (e.g., idle or busy), as well as other aspects such as the number of aircraft waiting to take off or land. Events might correspond to aircraft arrivals or departures.

A parallel discrete event simulation program can be viewed as a collection of sequential discrete event simulations that interact by exchanging timestamped messages. Each message represents an event that is scheduled (sent) by one simulator for another. Each sequential simulator is referred to as a logical process or LP. For example, a simulation of the global air traffic system could be constructed by creating a sequential simulation of each airport and allowing each simulator to schedule events (i.e., send messages) to other simulators. Messages transmitted between airport simulators might represent the arrival of an aircraft flying from one airport to another.

Much of the work in PDES has focused on the synchronization of PDES programs. The synchronization problem is concerned with ensuring that the execution of a PDES program will produce exactly, or in some cases approximately, the same results as a sequential execution of the same program where all events are processed one after the other in timestamp order. One can show that this can be achieved by ensuring that each LP processes events in timestamp order, including messages (or events; here we use the terms messages and events synonymously) received from other processors. Synchronization algorithms are broadly classified into two categories: conservative and optimistic. Conservative algorithms such as the Chandy/Misra/Bryant algorithm [Bryant 1977; Chandy and Misra 1978] use a mechanism that blocks the execution of an LP until it can guarantee that no event with a smaller timestamp will later be received. This requires specification of a lookahead value. If an LP is currently at simulation time $T$, and its lookahead value is $L$, then any message later sent by the LP must have a timestamp of at least $T+L$. One can associate lookahead values with links rather than LPs. All conservative algorithms rely on a sufficiently large lookahead value to achieve concurrent processing of events. Finally, within the class of conservative algorithms, some algorithms use global barrier synchronizations to manage the execution. Notable examples are the Bounded Lag [Lubachevsky 1989] and YAWNS [Nicol, Micheal et al. 1989] algorithms.

While conservative algorithms take precautions to ensure LPs always process events in timestamp order, optimistic algorithms allow events to be processed out of order, but use a rollback mechanism to recover from such errors. Time Warp [Jefferson 1985] was the first optimistic synchronization algorithm and remains the most widely used optimistic approach to this day. There is extensive literature in both conservative and optimistic algorithms. Much of this work is presented in greater detail in Fujimoto [2000, 2015].

\subsection{Distributed Simulation}

Distributed simulation is concerned with the execution of simulations over computing platforms that span a much broader geographic extent than parallel computers. In contrast to parallel simulations, where the processors executing the simulation reside 
within a cabinet inside a machine room, a distributed simulation may execute on a set of machines interconnected through a local area network, globally distributed computers communicating via the Internet, or predictive simulations embedded within a physical environment such as a sensor network monitoring traffic in a city. A central objective of utilizing distributed simulation systems has been to allow exploitation of geographically distributed resources such as equipment or people in the distributed simulation exercise, or executing simulations in close physical proximity to live data streams to predict future states of an operational system.

A central issue in and often the principal motivation for utilizing distributed simulation concerns the desire to integrate simulators operating on different computing equipment into a single simulation environment. Much of the early work in distributed simulation focused on training systems - tank simulators, flight simulators, computergenerated forces, and a variety of other models could be combined to create a distributed virtual environment into which personnel could be embedded to train for hypothetical scenarios and situations.

While synchronization, also known as time management in the distributed simulation literature, is also an issue in distributed simulation, it is not the only issue and typically is not the most important one. Much of the early research in distributed simulation focused on distributing information among the simulators participating in the distributed simulation in an efficient and timely manner. Much of this work was concerned with communication protocols such as multicast mechanisms or methods to reduce the amount of data that needed to be communicated through the use of techniques such as dead reckoning [Miller and Thorpe 1995] or data distribution management [Morse and Zyda 2001]. Because a large emphasis in distributed simulation has been to achieve interoperability among separately developed simulators, a substantial amount of effort has focused on developing standards to interconnect simulations. This has resulted in the Distributed Interactive Simulation (DIS) (IEEE Std 1278.1-1995 1995, IEEE Std 1278.2-1995 1995) and the High-Level Architecture (HLA) (IEEE Std 1516-2010 2010, IEEE Std 1516.1-2010 2010, IEEE Std 1516.2-2010 2010) standards. Many of the technical issues addressed by the distributed simulation research community are discussed in Fujimoto [2000] and Tolk [2012].

The remainder of this article is organized as follows. The next section briefly introduces six areas of current and future research in parallel and distributed simulation where additional work is required and argues why each of these areas is timely in light of current technological trends and applications. Section 3 forms the main body of this article. It discusses each of these six research areas in greater detail by proposing an overarching challenge in that area, discussing prior work, and highlighting key research issues and problems that have not yet been satisfactorily addressed. While some of these challenges are relevant only to PDES or distributed simulation, several span both areas. Finally, Section 5 provides concluding comments regarding the future of the parallel and distributed simulation field.

\section{KEY RESEARCH CHALLENGES}

The parallel and distributed simulation field has grown and evolved over the last four decades. Today, the field faces many new challenges that have arisen from needs emerging from new applications, on the one hand, to dramatic changes in the underlying hardware and software systems on which they operate, on the other. The six areas enumerated to follow define technical challenges yet to be addressed where significant advances could yield impacts spanning multiple application domains:

1. Application-driven, scalable simulations of large, complex networks. Much of the research to date concerning large-scale, massively parallel simulations has focused 
on demonstrating the capability of PDES technology on synthetic workloads. We advocate an application-driven approach demonstrating the ability of large-scale simulations to gain new insights into the behavior of real-world networks at scale. For example, one challenge is to create large-scale simulations of networks that have irregular topologies with skewed node degree distributions.

2. Exploitation of heterogeneous machine architectures using Graphics Processing Units (GPUs). GPUs are becoming common in platforms ranging from large-scale supercomputers to clusters and desktop machines. A growing body of research has been exploring exploitation of these devices for parallel discrete event simulation applications. If successful, research in this area may enable PDES to achieve new levels of performance for certain classes of problems.

3. Making parallel and distributed simulation broadly accessible through simpler model development and cloud computing platforms. The difficulty of developing efficient parallel simulation code remains a challenge, and techniques to simplify software development and maintenance are badly needed. Cloud computing offers the ability to make parallel and distributed simulation technology broadly accessible to virtually all users without incurring the expense of purchasing and operating high-performance computing platforms, a long-standing issue that has prevented widespread adoption of parallel and distributed simulation technology in the past.

4. Online decision making using real-time distributed simulation. The emergence of ubiquitous computing, wireless sensor networks, and vast amounts of data enable simulations to be embedded into operational systems at unprecedented scales in emerging areas such as smart cities. Distributed simulation can play a large role in online management and optimization of operational systems.

5. Energy-and power-efficient parallel and distributed simulation. Power and energy consumption have emerged as major issues in computing. It affects battery life in mobile computing platforms and is a major expense in data centers. Yet power and energy consumption has received very little attention in the parallel and distributed simulation field to date. Little is known concerning the power and energy characteristics of parallel and distributed simulations, or how to effectively manage these aspects of the program's execution.

6. Rapid composition of distributed simulations. Rapidly composing separately developed simulations remains a formidable challenge. Much progress has been made, for example, in creating technologies supporting software frameworks and standards. However, composing separately developed simulations for different purposes remains a time-consuming task, or may not even be possible. The importance of this area is increasing with the recognition that in many cases, one must use nonreductionist approaches to study systems as a whole rather than their constituent pieces in isolation.

We believe research in each of these areas is timely in light of recent technology trends and application needs. Key factors motivating the first three areas concern important technological changes in the underlying computing platform. This phenomenon is not new to the parallel and distributed simulation. Indeed, parallel discrete event simulation emerged with the availability of commercial multiprocessor systems, and distributed simulation emerged from advances in computer networking, among other technologies. Massively parallel supercomputers containing over a million cores, GPUs, and cloud computing have emerged in importance in the last decade, motivating research in parallel and distributed simulation to exploit these platforms.

Recent trends such as the Internet of Things and "Big Data" have strong implications concerning the future of parallel and distributed simulation. Online decision making is an area of increasing importance with the emergence of mobile computing and growth 
in technologies such as sensor networks. Parallel and distributed simulation is complementary to the exploitation of data analytics. While models derived purely from data analytics offer much potential to provide predictive capabilities in many situations, these models do not include behavioral descriptions of the system under investigation that are necessary for what-if experimentation, or analysis of situations where sufficient data or the right data are not available, for example, due to privacy or other concerns. At the same time, power and energy consumption has become an important consideration in computing, both for mobile computing platforms and data centers.

Finally, while composition of simulations has remained a long-standing problem in distributed simulation for many years, new application areas such as smart cities that call for modeling systems of systems highlight the growing importance of this technology. Much progress has been made in developing methodologies and standards to enable simulations to interoperate. However, we are far from achieving approaches where separately developed models can be easily composed in a "plug-and-play" fashion with some assurance that the resulting models are valid.

\section{SIX RESEARCH CHALLENGES}

In the following, we review in greater detail ongoing work in each of these areas, as well as key research challenges that are yet to be addressed. We begin discussion of each area with a challenge that attempts to encompass research in that area.

\subsection{Large-Scale Parallel Discrete Event Simulations of Complex Networks}

Challenge 1. Create scalable, realistic large-scale simulations of irregular systems such as complex networks and demonstrate their ability to create new insights into real-world systems.

At the heart of the first challenge is to create credible simulation models of realistic networks at scale in order to gain insight into their behavior. Over the years, numerous successes have been achieved demonstrating the capabilities of PDES technology to accelerate the execution of discrete event simulations. For example, Fujimoto et al. [2003] examined packet-level simulation of computer communication networks on supercomputers. The parallel simulator yielded performance as high as over 200 million events per second (eps) using a conservative synchronization algorithm executing on a supercomputer using 1,536 processors in 2003. By comparison, comparable simulators executing on a sequential machine yielded performance less than 200,000eps. In 2007, studies using a synthetic benchmark called PHOLD yielded performance exceeding 529 million eps using an optimistic synchronization algorithm on a 16,384-processor machine [Perumalla 2007], and in 2009, performance of 12.26 billion eps using 65,536 processors was achieved [Bauer et al. 2009], both using IBM Blue Gene machines. In 2013, Barnes et al. [2013] were able to achieve 504 billion eps using almost 2 million cores of a Blue Gene/Q machine. Studies of large-scale simulations of specific applications include epidemic spread [Bisset et al. 2009; Perumalla and Seal 2010; Perumalla et al. 2014] and electromagnetic signal propagation [Bauer et al. 2009].

It is instructive to examine the performance that was achieved for each core. For this purpose, we use the events/second metric because the studies cited later focus on applications with a modest amount of computation per event; this metric is less useful for applications that do not have this property. The studies reported in Fujimoto et al. [2003], Perumalla [2007], Bauer et al. [2009], and Barnes et al. [2013] yielded event rate performance per core of $138 \mathrm{~K}$ [2003], 32K [2007], 187K [2009], and 256K [2013] eps/core, respectively. This represents only a twofold improvement in singlecore performance over a span of 10 years. These data highlight that performance improvements today are being driven almost entirely by increases in parallelism. This is not surprising. Processor clock rates have seen only modest increases since 2005 due 
to physical constraints concerning heat dissipation, resulting in an explosion in the number of cores in supercomputer architectures since 2005. Throughout much of the 1990s and up until 2005, the most powerful supercomputers contained only thousands of cores. The most powerful machines today contain millions.

The studies just cited have, to a large extent, focused on a bottom-up, technologydriven approach. By this we mean these studies were focused on understanding the scalability and limitations of PDES technology itself, largely independent of the application. While important, an alternative top-down, problem-driven methodology focusing on specific application questions is needed. Such studies will focus on answering important questions in a specific problem domain and derive salient properties of these applications that call for new approaches to parallelization. Recent work in this direction for computer network simulation includes Kunz et al. [2016] and Jin and Nicol [2015].

To illustrate this point, consider the issue of network topology. The studies described earlier focused on synthetic network topologies that are highly regular such as a toroid, ring, or fully connected network. The PDES topology is a graph with each node representing an LP, and each link connecting two LPs indicating those LPs may communicate by sending a message between them. Here, we assume undirected arcs and do not distinguish between the sending and receiving LP. The benchmarking studies described earlier all assumed a regular network topology such as a toroid where the workload could be uniformly distributed over the network with little difficulty. In practice, few real-world applications exhibit such ideal properties.

A substantial amount of research in the past decade has focused on the topology of networks that arise in real-world applications. More often than not, the networks of practical interest are highly irregular. One class of topologies that have become known as scale-free networks [Barabasi and Albert 1999] has been observed to often arise. A scale-free network is one where the node degree follows a power-law distribution. A distinguishing characteristic of scale-free networks is that a significant number of nodes, referred to as $h u b$ nodes, contain a large node degree, while most nodes, often referred to as leaf nodes, contain a relatively small degree. Scale-free networks are clearly an example of networks with a skewed node degree distribution.

Scale-free networks have received a considerable amount of attention in recent years because it has been observed that many real-world systems contain networks that exhibit at least approximately the scale-free property [Wang and Chen 2003]. For example, it is widely believed that the autonomous system (AS)-level topology of the Internet is scale-free [Faloutsos et al. 1999; Siganos et al. 2003; Zhang et al. 2011]. In the broad area of systems biology, the study of complex biological systems, proteinprotein interaction networks have been demonstrated to follow scale-free distributions [Kitano 2002]. Some financial networks such as the interbank payment network exhibit scale-free behavior [Soramaki et al. 2007]. Social networks, the World Wide Web, the internal structuring of superconductors, the airline transportation network, and human interaction networks such as those characterizing the spread of diseases have been reported to exhibit the scale-free property to varying degrees.

Other networks, while not following a power-law distribution, do appear to have heavy skew in their node degree distribution. For example, initial studies suggested that the router-level topology of the Internet followed a power law, but more recent work reports that the topology is driven by engineering and economic considerations as well as physical node and link capacity constraints, resulting in a node degree distribution that is skewed but does not follow a power law [Li, Alderson et al. 2004]. The routerlevel topology contains many high-degree routers at the edge of the network designed to aggregate traffic from many low-bandwidth connections coupled with a mesh-like core of high-capacity routers with a smaller number of high-bandwidth links. 
The network topology has a large impact on the efficiency of parallel discrete event simulation techniques in terms of parallelism and overhead of the synchronization algorithm. For example, in Guclu et al. [2004], a network of LPs configured in a ring topology is augmented with one additional link per LP for synchronization purposes to create a small-world topology in order to improve scalability.

It has been observed empirically that the distribution of event-level parallelism in simulations of communication networks can lead to severe load imbalances [Liu and Chien 2004]. In Dobrescu et al. [2008], experiments with PDNS, a parallel version of the NS2 simulation [Riley et al. 2004] to simulate scale-free networks, reported some success in achieving speedups with a small number of processors but noted a slowdown for a modest level of simulated network traffic using only six processors. In D'Angelo and Ferretti [2009], load distribution issues in parallel scale-free network simulations are examined and evaluated in simulating gossip protocols. The relationship between power-law topology and parallel simulator performance was studied in Pienta and Fujimoto [2013], where it was observed through both analytical models and simulations that very large network simulations may yield very limited parallelism. For example, scale-free networks containing tens of thousands of network nodes often exhibit less than hundredfold parallelism. Finally, other work examines parallelism in the generation of large scale-free networks [Hruz et al. 2010; Yoo and Henderson 2010] and use of hybrid synchronization approaches using a combination of local and global synchronization techniques [Liu and Rong 2012].

Scale-free networks are a challenging test case for some PDES synchronization algorithms. One reason they are problematic is because scale-free networks possess the so-called small-world property. Small-world networks are distinguished by the fact that the average minimum path length between two randomly selected nodes in the network is very small; this length may be only a few hops even for networks containing millions of nodes. Power-law distributions are characterized by the parameter $\lambda$ that determines the number of high-degree nodes that will occur. In cases with $2 \leq \lambda \leq 3$, a common range for natural scale-free networks, the average path length grows very slowly, log $\log N$ for networks containing $\mathrm{N}$ nodes [Cohen and Havlin 2003]. Stated another way, the number of nodes (LPs) reachable from a given node increases exponentially with the number of hops. This suggests that algorithms that require examination of other LPs to determine what events are safe to process are doomed to failure because a very large number of nodes will have to be examined to determine what events are safe. Windowing schemes such as that used in the Bounded Lag Algorithm [Lubachevsky 1989] are unlikely to solve this problem because the relevant "neighborhood" of nodes that must be examined grows too rapidly.

Network topology may also impact the performance of optimistic synchronization algorithms. The propagation of rollbacks through the network is dependent on network topology. The relationship of the rollback propagation mechanism to topology is complex, and not well understood; however, it seems unlikely that optimistic execution, by itself, can achieve scalable performance for large numbers of processors. Thus, the limited amount of parallelism in these networks coupled with the reliance on massive parallelism to achieve high performance on modern supercomputers makes exploitation of PDES challenging for many real-world applications.

To illustrate this point, Figure 1 shows the maximum amount of speedup available in simulations of scale-free network assuming an ideal parallel simulator with no overheads for communications and synchronization [Pienta and Fujimoto 2013]. The node degree distribution follows a power law where the node degree probability is $c k^{-\lambda}$, where $k$ is the node degree and $c$ is a normalization constant. Smaller values of $\lambda$ yield heavier tails in the distribution, that is, a higher probability of hub nodes. As can be 


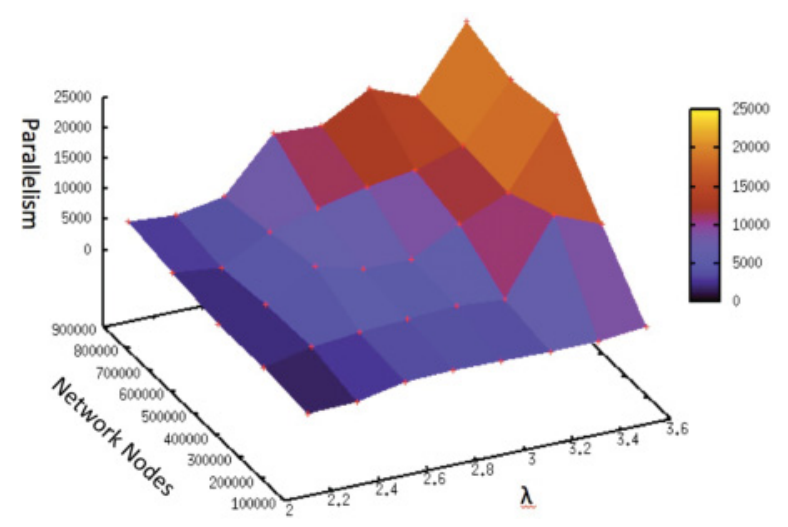

Fig. 1. Available parallelism of scale-free network simulations.

seen, the potential speedup is much less than the size of the network, due to bottlenecks arising at hub nodes.

While these observations highlight one important challenge for PDES technology, more broadly we advocate a problem-driven methodology. Such a methodology focuses on defining specific domain challenges that require large-scale simulations and derives parallel and distributed simulation challenges motivated by these applications. In many instances, solutions will be applicable to multiple domains.

Finally, we note other issues related to scalable execution that span multiple application domains. Fault tolerance increases in importance as the increasing number of cores reduces the mean time between failures. Large-scale simulations can have large-scale data requirements and produce massive amounts of output data, raising questions of I/O performance. Virtually all usage scenarios will call for multiple simulation runs (e.g., see Yau [1999]); techniques to improve the efficiency of multiple replicated runs, for example, by exploiting commonalities among these runs, are needed.

\subsection{Exploiting Graphics Processing Units in Parallel and Distributed Simulation}

Challenge 2. Develop practical, scalable techniques to exploit graphical processing unit (GPU) accelerators in modern high-performance computing and mobile computing platforms to significantly accelerate real-world simulation applications.

Modern supercomputers are increasingly making use of hardware accelerators to achieve increased parallelism and higher levels of performance, particularly for dataparallel computations. GPUs are similarly appearing in smartphones and mobile computing devices, providing increased computational power. A GPU is a hardware accelerator that offloads computational tasks from the central processing unit (CPU). Initially designed to render graphics in workstations and personal computers, they have since found broader application as a means to accelerate data-intensive numerical computations. The combination of low per-unit cost resulting from high volume production and their suitability for numerical computing has made GPUs an attractive component for high-performance computing and mobile systems. Much of the work to date has focused on exploiting GPUs for numerical computing problems. However, a growing body of work has focused on discrete simulations, although much of this work to date has focused on time-stepped simulation applications such as cellular automata rather than event-driven simulation.

Many of the challenges associated with exploiting GPUs stem from their distinctive hardware architecture. Effective exploitation requires one to develop an approach 


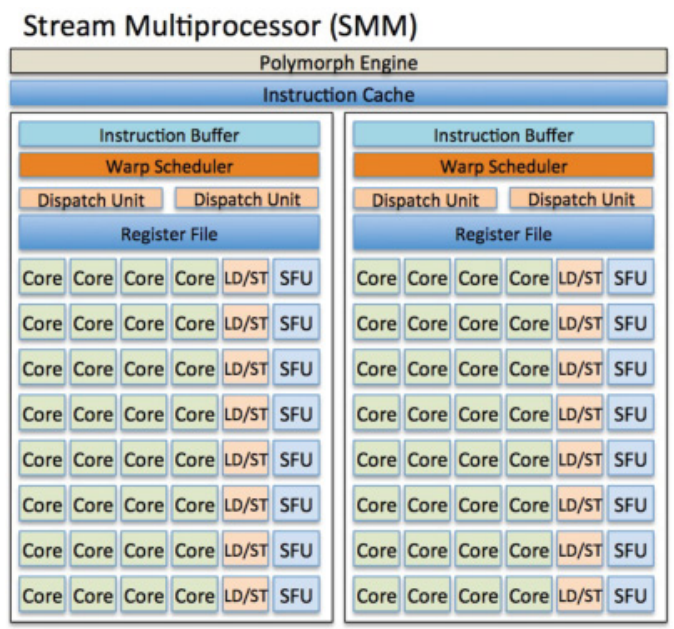

Fig. 2. Computation core of the NVIDIA GM107 Maxwell architecture [NVIDIA 2014]. Half of the cores of a single stream multiprocessor are depicted.

to map the PDES computation to the hardware in a way to effectively exploit the GPU. The main challenges that must be addressed may be classified as relating to the mapping of the PDES computation to the computational, memory, and communication architectures of the GPU:

1. Computation architecture: The discrete event simulation computation must be mapped to the Single-Program, Multiple Data (SPMD) stream style of parallel execution used in GPUs.

2. Memory architecture: The irregular, dynamic data structures typically used in discrete event simulations must be mapped to the GPUs' memory architecture, which is better suited for static, regular data structures such as matrices and arrays.

3. Communication architecture: Communication bandwidth for transmitting data to and from the GPU is limited; copying data between the CPU and GPU memory can be time consuming and must be kept to a manageable level to avoid severe performance degradations.

Each of these issues is discussed next.

3.2.1. SPMD Execution. For the purpose of this discussion, we will use the NVIDIA GPU architecture that is widely used in HPC platforms today as a reference. A portion of the GM107 "Maxwell" architecture is shown in Figure 2. The GPU operates as a coprocessor that performs computations concurrently with a general-purpose CPU. Each GPU includes several stream multiprocessors (SMMs) such as the one shown in this figure. Each SMM uses an SPMD mode of execution where the same program is executed concurrently across multiple data elements. Specifically, each SMM includes 128 computing cores (also referred to as CUDA cores or stream processors), where each core has roughly the capability of an arithmetic logic unit (ALU). Each SMM executes computational units known as warps (no relationship to Time Warp), where each warp consists of a set of 32 threads of execution. A common instruction can be executed concurrently by all 32 threads, each operating on different data elements.

From the programmer's perspective, the GPU computation is organized as a set of thread blocks, where each thread block consists of one or more warps. A kernel grid is a collection of thread blocks that are invoked by the CPU. Each thread block is assigned 
to a single SMM for execution. Each SMM can support up to 2,048 concurrent threads, or 64 warps. The 32-thread warp is the unit of computation that can be scheduled for execution, and as noted earlier, each warp executes as an SPMD program.

The computation of a discrete event simulation can be viewed as a set of individual event computations, where each event computation may (1) modify one or more state variables and/or (2) schedule new events. The SPMD paradigm used by the GPU implies the simulation would best be structured as containing a small number of different types of events, ideally only one. For example, a queueing network simulation utilizing a firstcome-first-serve (FCFS) discipline could be programmed with two event types: a job departure and a job arrival event. However, if the time to move a job from a server to another queue is zero, one observes that the departure of a job from one queue occurs at exactly the same simulation time as that job's arrival at the next queue. Thus, the job's departure and arrival events could be combined into one event, for example, by incorporating the computation for the departure event into the arrival event corresponding to the job arriving at the next queue. Alternatively, if a first-comefirst-serve queueing discipline is used, one can eliminate the departure event entirely, and only process arrival events. Modifications such as these reduce the number of different event types, making the program better suited for SPMD processing.

Restructuring the program to reduce the number of different types of events is not sufficient to ensure efficient execution. Data-dependent conditional statements within a single event procedure can cause different event computations (threads) to execute different sequences of machine instructions. In other words, some LPs executing the predicate of the conditional based on one set of data may take the "then" clause of an if-then-else statement, while others utilizing different data on the same predicate may execute the "else" clause. The execution of these different sequences of instructions must be performed sequentially in SPMD architectures. Therefore, one should also minimize or eliminate statements within the program with data-dependent conditional control flows. This may be difficult or impossible for many complex simulation applications.

Work examining the execution of PDES programs on SPMD architectures date back to the late 1980s. Early work includes examining SPMD architectures for queueing network and Ising spin simulations [Lubachevsky 1989] and logic simulation of electronic circuits [Chung and Chung 1991]. Some work has addressed this problem in the context of GPU architectures. For example, in Kunz et al. [2012], an approach is described that involves sorting events according to event type and clustering their execution to address the problems arising from SPMD execution described earlier.

3.2.2. Irregular Data Structures. A second challenge concerns the mapping of data structures used by PDES programs to the memory hierarchy provided in modern GPU architectures. The NVIDIA GPU discussed earlier includes a global memory that is typically a few hundred megabytes to a few gigabytes that can be accessed by any thread in the SMM. Access to global memory is relatively slow with a throughput of a few hundred gigabytes per second; however, the GPU will optimize accesses to consecutive memory locations by aggregating the individual references into a single operation. There is also a per-block memory accessible only to the threads within a block that is a few tens of kilobytes and that offers seven times the bandwidth of the global memory. The per-block memory is implemented as a set of $\mathrm{N}$ memory banks. Multiple threads can concurrently access different banks of the memory, but accesses to values within a single memory bank must be serialized. Therefore, care must be taken to assign data to memory banks in order to maximize performance. For example, if the elements of a row of a two-dimensional matrix are mapped to the different memory banks while elements of a column are mapped to the same bank, arithmetic operations operating 
on a row of the matrix can be executed efficiently, while those operating on a single column will result in memory contention and serialized execution.

Two key challenges in exploiting GPUs for PDES computations concerns memory allocation and the mapping of data structures to the GPU's memory. GPUs are best suited for regular computations operating on static vectors or matrices of data. However, traditional PDES programs partition the state of the simulation among a set of LPs, and each LP is viewed as an autonomous component of the simulation rather than arrays of data. This can lead to irregular accesses to memory elements depending on which LPs execute when, and how the LPs memories are mapped to the GPU's memory.

Further, the NVIDIA GPU does not support dynamic memory allocation, whereas dynamic memory allocation is common in many PDES applications. This can be managed by utilizing statically allocated arrays for storage and array indices rather than pointers. However, unless successive references to arrays utilize consecutive memory locations, it will be difficult to exploit GPU optimizations such as coalescing memory references, and collisions to memory banks may lead to performance degradations.

3.2.3. Communication and Memory Copies. In systems such as the NVIDIA GPU described earlier, CPU and GPU memory occupy separate address spaces; the CPU cannot directly access the GPU's memory and vice versa. It is often the case that the GPU memory is not large enough to hold the entire computation, necessitating the movement of data between CPU and GPU memory throughout the execution of the simulation. Communications between the two memories is time consuming. This limitation may be addressed in future architectures that support shared memory between the CPU and GPU; however, for now, one must structure the program so copies between CPU and GPU memory occur sufficiently infrequently to avoid significant performance degradations. This highlights the need for a large amount of simulation computation to be performed between memory copy operations.

Variations of this problem have arisen in computer architectures long before the invention of GPUs-a similar problem arises in cache memory systems where data must be transferred to the cache before the CPU can operate on the data. A common approach to addressing the cache memory problem is to hide the latency associated with transferring data by allowing the CPU to perform other computations while the data transfer is taking place. A similar approach can be applied here to address the problem of latency for memory transfers between the CPU and GPU memory. Ideally, GPU computations may proceed concurrently with data transfers to and from the GPU memory.

An approach to latency hiding for PDES codes executing on GPUs is discussed in Kunz et al. [2012]. Consider a set of event computations that are independent and can be executed concurrently. An approach using pipelining is used to hide the latency of data transfers between CPU and GPU memory. The pipeline consists of three stages. The first involves transferring data from the CPU to the GPU memory, the second involves performing event computations within the GPU, and the third involves transferring the results of these computations back to CPU memory. If there are many concurrent events and the event computations involve sufficient computation so that the GPUs never have to wait for data transfers to complete, this approach can achieve full utilization of the GPU hardware for concurrent event processing, assuming of course the event computation is well structured as an SPMD program, as discussed earlier. The overall approach described in Kunz et al. [2012] also exploits parallelisms resulting from multiple simulation replications to reduce the time required to perform multiple independent simulation runs.

Scheduling and load balancing are other important issues that arise in heterogeneous computer architectures. One approach to address this issue is presented in Romdhanne 
et al. [2013]. A scheduler is proposed that is based on a hybrid style using both timedriven and event-driven execution. A dynamic mechanism is also proposed to maximize the flow of event processing.

Supercomputers today often include many processing nodes, each equipped with multiple CPUs and GPU accelerators connected via a high-speed network. These machines introduce additional complexity for large-scale parallel codes, further exasperating the issues described earlier. Latency hiding techniques become even more important in exploiting these computing architectures [Zou et al. 2013]. Effective exploitation of cache memory and managing large numbers of computing threads are similarly critical in many-core architectures using large numbers of cores in the MIMD computing model.

3.2.4. Applications. Much of the work to date using GPUs for discrete simulations has focused on time-stepped models. Cellular automata are well suited to the constraints imposed by GPU architectures. Each thread can capture the behavior of a single cell. Because the cells execute the same rules, the computation is well suited for SPMD computation provided the rules do not require complex data-dependent conditional behaviors. The state of the simulation can be implemented using a static array data structure, and the fact that each cell only interacts with neighboring cells leads to a well-structured, regular, memory reference pattern. Further, provided the state of the simulation fits within the GPU's memory, one need not copy state between the CPU's and GPU's memories except at the beginning and end of the simulation. Use of GPUs for time-stepped simulations have been explored in applications such as traffic simulation [Perumalla et al. 2009; Xu et al. 2014], systems biology [Falk et al. 2011], and Ising Spin [Hawick et al. 2011], among others.

A limited amount of research has focused on event-driven simulation execution. An approach to executing queueing network simulations on GPUs is described in Park and Fishwick [2011]. This approach effectively uses a time-stepped execution mechanism to implement the discrete event simulation. The approach introduces errors in the computation by forcing event timestamps onto time-step boundaries, introducing tradeoffs between accuracy and execution efficiency. A 10-fold speedup was achieved with small errors, although it was observed that the error increases with the size of the cluster. Another approach utilizing a three-step approach-generate events, sort them, process them-and use of time-parallel simulation techniques is described in $\mathrm{Li}$ et al. [2013].

Finally, another body of research has focused on hybrid approaches where portions of the simulation are executed on the GPU while the remainder of the simulation executes on the CPU. For example, in Liu et al. [2014], a method for hybrid simulation of telecommunication networks is described where a discrete event packet-level simulation is performed by the CPU while a continuous numerical fluid flow simulation of traffic is executed by the GPU. Approaches such as these involve partitioning the computation into separate tasks and mapping each to the architecture best suited for that task.

3.2.5. Future Directions. While there is a growing body of work concerning PDES exploiting GPU hardware accelerators, the use of these architectures is far from common, and much work remains to be done. It should be clear from the previous discussion that mapping a PDES computation onto parallel computer architectures using GPU accelerators is not a simple task. Many low-level details of the architecture must be taken into consideration in developing the PDES code. Further, as these architectures change and evolve over time, a significant effort may be required to recode the application as architectures evolve. One approach to addressing this problem is to develop new simulation languages that simplify code development, and compilers that automate details concerning the mapping of the simulation code to the underlying architecture. While 
languages such as CUDA exist, they are not designed with simulation computations in mind. The discrete event simulation field has a long and successful history of developing simulation languages, many of which are widely used in industry. Given this tradition, languages designed to generate efficient PDES codes for heterogeneous parallel computers have the potential to make advanced computer architectures more broadly accessible to the general simulation community.

Moreover, as noted earlier, the bulk of the work to date concerning use of GPUs for discrete-time simulations has focused on time-stepped simulations; by comparison, relatively little work to date has focused on event-driven simulations. Additional work in addressing effective exploitation of GPUs for event-driven computations is needed.

\subsection{Making Parallel and Distributed Simulation Widely Accessible: Simplifying Model Development and Exploitation of Cloud Computing}

Challenge 3. Enable parallel and distributed simulation technologies to become broadly accessible to the general modeling and simulation community by simplifying the development of simulation models and exploitation of cloud computing.

The lasting impact of parallel and distributed technologies will come from its use to solve real-world problems faster and/or more economically than would otherwise be possible. While many technical successes have been reported in the literature using parallel simulation, the vast majority of simulations that are developed today use sequential execution. A long-standing problem faced by the parallel and distributed simulation community has concerned how to make the technology more broadly accessible to the general modeling and simulation community. One issue concerns the complexities associated with creating parallel and distributed simulation models. A second concern is gaining access to suitable high-performance computing platforms. Each of these issues is discussed next.

3.3.1. Simplifying the Development of PDES Codes. Simplifying the creation of PDES codes has long been recognized as an important challenge in the parallel and distributed simulation community. In Fujimoto [1993], several challenges that stood in the way of widespread adoption of PDES technology were discussed. Four avenues to address this issue were proposed: (1) creation of application-specific libraries to hide the details of parallel simulation software development from users; (2) new parallel simulation languages to simplify the development of parallel simulation codes; (3) support for shared state, also to simplify software development; and (4) development of methods to automate the parallelization of sequential simulation codes. To date, progress has been made in all four areas, but arguably, the greatest impact has been in areas (1) and (4).

The first approach, use of application libraries, is perhaps best exemplified by Qualnet [Scalable Network Technologies 2012], a commercial simulation tool for modeling telecommunication networks. Parallel libraries of simulation components enable one to configure a simulation from a graphical user interface while hiding the details of parallel execution in an underlying simulation executive. There is little question that hiding the many complexities associated with developing and executing parallel simulations from users is essential for widespread adoption by a large user community. This approach offers the ability to hide parallel processing issues from the user, but requires at least some simulation model developers to have expertise in parallel computing.

Automated or semiautomated parallelization is another approach that has seen some success. This can be achieved through a "self-federating" approach. Here, multiple instantiations of a single sequential simulation are created and interconnected through a simulation executive or runtime infrastructure (RTI) software that provides at a minimum communication and synchronization services. Each sequential simulation models 
a portion of the overall system and is written largely as a sequential simulation program. This has emerged as a practical approach to realizing parallel simulations. For example, each sequential simulation might model a subnetwork of a large telecommunications system. Examples of this approach are described in Nicol and Heidelberger [1996] and Riley et al. [2004], among others. Because this approach effectively involves federating a sequential simulation with itself in a parallel or distributed computing environment, it avoids many of the interoperability issues that arise when federating different simulators, as will be discussed later. While not all sequential simulators are amenable to this approach, experience has shown that this can be a viable approach to parallelization.

Based on self-federation, a practical approach to creating PDES codes is to develop modular sequential simulations with a few modest guidelines such as avoiding the use of global variables. If conservative synchronization is used, one must develop an approach to define lookahead within the simulation in order to allow efficient parallel execution. If optimistic synchronization is used, an approach to incorporate rollback into the simulation code is required. One approach based on the HLA standard defines a time management converter coupled with a mechanism utilizing dirty bits of memory pages that are set by the operating system to mark modified pages of memory to implement state saving [Santoro and Quaglia 2012]. Mechanisms to allow access to shared state variables among LPs are described in Pellegrini et al. [2012]. Another approach is to use a reverse compiler to automatically generate code to implement rollback [Vulov et al. 2011]. Creation of effective, practical reverse compilers is currently an open area of research.

New modeling languages coupled with mechanisms to automatically translate modeling abstractions to efficient parallel simulation code are another important avenue for future research. Modeling complex phenomena is challenging in its own right even without introducing the complexities of concurrent model execution. A large gap exists between the model residing in the mind of the modeler and detailed parallel simulation code. Suitable intermediate representations, sometimes called conceptual models, are needed to provide descriptive yet precise specifications of model state and behavior. For example, well-known abstractions in the classical simulation literature include queueing networks, Petri nets, and cellular automata. Optimizing compilers are needed to automate the translation of specifications using these abstractions into efficient parallel simulation code.

Simulations today are often developed by a team of researchers, each with expertise in a different field. Another approach to facilitate model development is to create application-specific simulation languages that provide constructs and abstraction familiar to domain experts but hide the details associated with parallel execution. Important issues such as partitioning the simulation model to execute on different processors, load distribution, and synchronization must be handled automatically and transparently from the perspective of the simulation model developer. Effective debugging environments must be readily available. Output analysis must be no more difficult than that corresponding to sequential execution. This approach has not been widely studied by the parallel and distributed simulation research community and represents another direction for future research.

3.3.2. Exploitation of Cloud Computing Environments. A significant impediment limiting widespread exploitation of PDES technology for large-scale simulation applications has been the need to have access to suitable high-performance computing machines. Cloud computing offers an approach to help address this issue. Cloud computing services such as Amazon Web Services' Elastic Compute Cloud (EC2), Microsoft's Azure platform, and Google's AppEngine provide virtualized hardware and software that can be 
accessed via the web. The cloud lowers the barrier to gaining access to high-performance computing machines. The cloud's "pay-as-you-go" economic model eliminates the need to purchase, operate, and maintain high-performance computing equipment locally [Fujimoto et al. 2010]. Further, by providing parallel and distributed simulation software as a service, cloud computing offers the ability to hide many of the complications of executing parallel and distributed simulation codes from the user, offering the potential to make exploitation of this technology less risky than is the case today.

Cloud computing is built upon a computational technique called virtualization. Briefly, virtualization is a concept dating back to the 1960 s where a computing environment for an application program is created that includes virtual system resources (CPU, memory, operating system, etc.) that are dedicated to the application program. The virtual resources are mapped to physical resources and managed by the underlying operating system. Virtualization provides a means to separate independent programs executing on a common hardware platform so that they do not interfere with each other, clearly essential for programs executing in the cloud.

Exploitation of the cloud for parallel and distributed simulation applications introduces new challenges. First, virtualization and execution on large distributed data centers on which public cloud computing environments are typically based may introduce certain computational and communication overheads that can introduce efficiency concerns, especially for communications. Second, the underlying computation resources are shared by many users, resulting in much greater variability in resource allocations throughout the execution of the simulation code. Third, new cost metrics may be needed with the pay-as-you-go model used in cloud computing. Finally, the cloud presents additional challenges for interactive simulations and those with real-time constraints. Each of these is discussed next.

Benchmarking studies of parallel scientific programs in public cloud environments such as Amazon's EC2 observed that parallel scientific codes ran significantly slower compared to execution on dedicated nodes of a cluster [Walker 2008; Ekanayake and Fox 2009]. Part of the reason for this is that cloud environments are typically optimized for high-bandwidth communications among applications rather than low latency for parallel computing applications that often exchange many small messages. High delay variance was also observed [Walker 2008]. This is problematic for many PDES codes that are accustomed to sending many small messages requiring quick delivery rather than fewer large messages requiring high bandwidth alone. Moreover, these studies suggest that techniques to hide communication latency should provide benefit for PDES codes executing on cloud environments. By its nature, optimistic execution offers some resilience to communication delays by allowing other computations to proceed while waiting for messages to arrive to a greater extent than conservative synchronization algorithms.

The situation for private clouds where the configuration can be more easily tailored to the user's needs differs from that of public clouds. An empirical study of PDES codes executing in cloud environments indicates that a PDES code executing on virtual machines can achieve performance comparable to a native execution [Yoginath and Perumalla 2013], suggesting that the virtual machine interface itself need not contribute a significant overhead. There is empirical evidence that execution can be almost as efficient as that on a native machine for private clouds [He et al. 2012; Jung et al. 2014]. Another study of adapting RTI software to the cloud reports some performance degradation, however [Feng et al. 2010].

A second major issue in cloud computing environments concerns resource sharing. Computing, communications, and I/O resources are shared among many users. Individual users are not guaranteed exclusive access to the processors assigned to that user's virtual cluster. This creates a significant amount of uncertainty and variability 
in the computational environment on which the parallel or distributed simulation code executes. This variability is cited as a likely cause of performance degradations of conservatively synchronized PDES codes executing on EC2 [Vanmechelen et al. 2012]. This can lead to difficulties for parallel simulation applications, especially those that utilize optimistic synchronization techniques. Architectures designed to provide flexible resource sharing are described in He et al. [2012], Liu et al. [2012], and Li et al. [2013].

The unpredictability of the underlying execution environment suggests that adaptive execution approaches may provide benefit for parallel and distributed simulations executing in the cloud. By this we mean mechanisms where a simulation code can change and adapt its behavior during the execution to provide resilience to changes in resources allocated during its execution. For example, dynamic load distribution mechanisms such as those described in Carothers and Fujimoto [2000], Boukerche and De Grande [2009], and Park and Fujimoto [2012] were designed to provide adaptation as the number of processors and amount of computation time allocated on individual processors varied over time. An optimistic synchronization algorithm may be especially prone to undesirable effects as the resources allocated to the computation vary because this can lead to an excessive amount of rolled-back computation [Malik et al. 2010]. Dynamic partition is proposed in D'Angelo [2011] to increase adaptation of parallel and distributed simulations executing on cloud platforms. Methods using relaxed synchronization techniques (e.g., allowing some events to be processed out of timestamp order) may offer another approach to improving efficiency on cloud computing platforms.

The economic model for cloud computing differs from that used in conventional parallel and distributed computing. Specifically, charges are based on the amount of computing resources that are consumed. This model may be less favorable for optimistic synchronization algorithms in the sense that one must "pay" for computation, even if it is later rolled back. This suggests that new metrics other than speedup may be required for parallel and distributed simulations executing in cloud environments. Different cloud computing vendors provide different pricing options for utilizing their service that may impact the approach that is most cost effective for a specific cloud computing platform.

Finally, cloud computing platforms may suggest a batch mode of processing where computations are sent to the cloud for execution and results are later returned when the jobs have completed. Another important potential use of the cloud is to execute interactive simulations where users, or as described momentarily, other devices may interact with a cloud-based parallel or distributed simulation during its execution. This suggests that hard or soft deadlines may be imposed on the execution. More research is required that considers the suitability of cloud computing for parallel and distributed simulations requiring such interaction. The effectiveness of fault tolerance mechanisms used in cloud computing environments is still another area requiring further study.

\subsection{Creating Reliable, Scalable Distributed Simulations for Real-Time Decision Making}

Challenge 4. Create online parallel and distributed simulation technologies suitable for real-time decision making utilizing live data feeds from sensors and other sources.

An emerging concept in computing called the "third platform" refers to the confluence of four technologies: cloud computing, mobile computing, big data, and social media. It has long been the case that the number of embedded and mobile computing devices dwarfs the number of traditional computers such as desktop machines and servers. The gap is widening rapidly with the explosion of interconnected, embedded computing devices, also known as the Internet of Things. The third platform is widely believed to be as disruptive an innovation as its predecessors, the first (mainframe) and second (distributed or client/server) platforms in the computing industry with transformative impacts in society. 
An area of growing interest concerns utilization of third platform technologies for real-time predictive analytics to manage and optimize systems to improve efficiency or increase resilience by enabling adaptation to changing circumstances. Embedded online simulations offer the potential to realize self-organizing, self-optimizing distributed systems. Parallel and distributed simulation can play a critical role in realizing such systems. Briefly, live data streams are used to capture the current state of the system and drive simulations in order to predict future system states or capture system dynamics. The predictive simulations may trigger modifications to the configuration of a physical system in response to events. For example, a sudden increase in traffic volume may indicate that the changes in traffic signal plans for the transportation system are necessary to help reduce congestion. Examples include vehicular/air traffic, computer systems, communication networks, supply chains, and many others that involve distributing limited resources/services among users. Since most of these are time-varying systems, transient-state analysis is important to the success of corresponding applications.

Dynamic Data-Driven Application Systems (DDDASs) [Darema 2004] are computational systems that dynamically incorporate data from a physical system into executing computations, thereby providing the ability of the application to dynamically steer the measurement process. A related concept known as symbiotic simulations emphasizes the inherent mutual benefits derived by the physical system and computational simulations through this interaction [Fujimoto et al. 2002; Aydt et al. 2009]. Such systems use networks of fixed and/or mobile sensors, possibly coupled with the use of crowdsourced data to develop a model of the current state of the system. Simulations then utilize this captured state to project future states and perform what-if analyses, often in conjunction with other optimization tools and techniques to inform operators as to how to improve system performance or reconfigure the sensor network.

These approaches have been widely studied and applied to various science and engineering disciplines for myriad purposes. One typical application concerns system monitoring, such as examining the structural and material health [Cortial et al. 2007], tracking wildfires [Douglas et al. 2006; Brun et al. 2012; Mandel et al. 2012] and hurricanes [Allen 2007], or prediction and tracking regional scale weather phenomena [Plale et al. 2005]. A second use concerns optimizing the operations of a physical system. For example, in an emergency situation, alternate evacuation scenarios may be modeled and evaluated in order to minimize evacuation time when unexpected events occur [Chaturvedi et al. 2006]. Additional examples include path planning for unmanned aerial vehicles [Kamrani and Ayani 2007; Madey et al. 2012], tuning computer networks [Ye et al. 2008; Erazo et al. 2015], managing semiconductor manufacturing systems [Low et al. 2005], and managing surface transportation systems to mitigate congestion [Suh et al. 2014]. They have been proposed for decision support systems for manufacturing applications, as described in Lendermann et al. [2005]. An approach to distributed, online simulations of queueing networks is discussed in Huang et al. [2010].

Online analysis for real-time decision making requires automation of many steps in the modeling and simulation life cycle in order to produce output results and recommendations in a timely fashion. It is instructive to consider the full life cycle of a modeling and simulation study in the context of online decision making. This life cycle typically entails (1) formulating the problem, (2) developing the conceptual model, (3) collecting and analyzing input data, (4) creating the simulation model, (5) verifying and validating the simulation model, (6) developing and executing an experimental design plan, and (7) analyzing results and developing conclusions. In this context, automation of steps 3 through 7 would provide substantial benefit. Automated input data collection and analysis requires at a minimum "cleaning" the data, or creating 
simulations that are robust and can tolerate some errors or missing values in the data stream. Machine-learning algorithms and data analytics may be needed to transform massive amounts of streaming data into useful information represented in a form that can be used to drive simulations. Further, data concerning the current state of the simulation can be used to validate and calibrate the simulation by comparing prior model predictions with reality. Creation and configuration of simulations and formulation of alternate scenarios must be automated in part or in whole, as well as execution of the simulations including replicated runs that may be required. Finally, analysis of the results produced by the simulation must be automated to enable timely decision making. Machine-learning and data analytics may also be applied here to assist in this process.

The mapping of distributed simulations to third-generation computing platforms requires further exploration. The traditional approach entails operating the simulations centrally (e.g., within the cloud). Current mobile applications such as Apple's iCloud service are thin clients where any significant computations are performed remotely in the cloud. Such applications require a relatively high-bandwidth Internet connection. Further, remote communication consumes more energy than local communications, an important consideration for mobile devices operating from battery power. Local WiFi bandwidth is typically $400 \mathrm{Mbits}$ per second, compared to $2 \mathrm{Mbits}$ per second for remote Internet access, and incurs much less latency; while bandwidth can be expected to increase in the future, latency improvements will not keep pace [Satyanarayanan et al. 2009].

An alternative approach is to embed the simulation computations within the physical system itself rather than relying on remote computing in the cloud. This approach is clearly needed when the simulations are used in tight control loops where the added latency of communications to a centralized cloud resource prevents rapid response to changing data. Executing distributed simulations in embedded devices enables the simulations to operate on detailed, disaggregated data that might be prohibitively expensive to transmit to the cloud in disaggregated form. An approach termed ad hoc distributed simulation utilizes this approach by focusing on simulations embedded within the physical system that operate on locally collected sensor data to predict future system states for transportation systems and queueing networks [Fujimoto et al. 2007; Huang et al. 2010].

Third-generation computing platforms present new challenges for the execution of distributed simulations. Interconnectivity among the components of the distributed simulation may be intermittent. In many cases, communications bandwidth will be limited and high rates of errors can occur. Distributed simulations must be robust and able to operate despite intermittent and unreliable data communications. Communications delay and jitter may be large in wireless and mobile platforms compared to traditional distributed computing systems, suggesting greater autonomy than is usually present in conventional parallel and distributed simulations, a factor motivating the ad hoc distributed simulation approach described earlier. Power consumption is a pressing concern, as will be discussed next. Finally, security (e.g., the ability of distributed simulations to produce reliable results despite false or malicious data) will increase in importance as systems are deployed.

\subsection{Understanding and Reducing Energy and Power Consumption in Parallel and Distributed Simulations}

Challenge 5. Understand the power and energy consumed by parallel and distributed simulations and develop means to adapt the simulation to optimally manage their use subject to constraints imposed by the environment in which it executes. 
Energy consumption has become a major concern for many parallel and distributed computing applications. The need to reduce energy consumption is clear in mobile and embedded computing, where reduced energy consumption can increase battery life or enable the use of smaller batteries, thereby reducing the size and weight of devices. In high-end computing, power consumption is a dominant cost associated with operating large data centers and supercomputers, and a substantial amount of effort has gone into developing techniques to reduce these costs. As discussed earlier, energy consumption has become the limiting factor preventing substantial further improvements in clock speed that dictate sequential computer performance. Despite the importance of power in computation today, very little attention to date has focused on understanding and developing techniques to minimize energy consumption in parallel and distributed simulations.

Energy is the capacity of a system to perform work. It is typically measured in units called joules, where 1 joule is the work done by an electrical circuit to move a charge of 1 coulomb through an electrical potential difference of 1 volt. Power is the amount of energy consumed per unit time, with 1 watt of power defined as the expenditure of 1 joule of energy per second. Minimizing energy usage and power consumption is not the same [Unsal 2008]. For example, decreasing the clock rate of the processor can lead to less power consumption. However, this will usually lead to a longer execution time, which can lead to an increase in the total amount of energy needed to complete the computation. Battery-operated devices are energy-constrained systems because they operate with a finite amount of energy; thus, a design goal might be to minimize the amount of energy utilized by the computation. On the other hand, in power-constrained systems such as supercomputers and data centers, the amount of energy may be less critical, but a design goal may be to reduce power consumption as much as possible or to some maximum level. Power-aware and energy-aware systems are those where power or energy consumption are a principal design consideration, and may include techniques to change the system's behavior based on considerations such as the level of energy being consumed or the amount of energy remaining in batteries. Finally, it may be noted that minimizing execution time does not necessarily minimize energy consumption. Energy consumption is affected by many factors, for example, the operation of the memory system, arithmetic circuits, and, most importantly, communication.

There is substantial literature in power- and energy-aware computing systems, and a variety of techniques may be employed. Dynamic voltage and frequency scaling (DVFS) is concerned with altering the voltage and/or clock speed by taking into consideration energy and performance constraints (e.g., see Hua and Qu [2003], Curtis-Maury et al. [2008], and Lively et al. [2014]). Dynamic power consumption in CMOS circuits is proportional to $F V^{2}$, where $F$ is the frequency at which the circuit is clocked, and $V$ is the power supply voltage. Several commercial microprocessors support modification of the processor's frequency and voltage to trade off power consumption and performance. Scheduling algorithms for embedded systems have been designed to balance energy savings with meeting real-time deadlines (e.g., see Quan and $\mathrm{Hu}$ [2001] and Cho et al. [2011]). Processors also commonly provide different modes of operation that utilize different amounts of power. Other work examines utilization of these modes of operation, sometimes in conjunction with DVFS [Niu and Quan 2004; Bhatti et al. 2010]. Other work includes predictive modeling of energy and power [Jia-Wei and Kung 1981; Hill and Marty 2008; Czechowski and Vuduc 2013] and embedded systems evaluations [Janapa Reddi et al. 2010; Fürlinger et al. 2011; Stanisic et al. 2013; Grasso et al. 2014].

To date, work in power- and energy-aware computing has largely focused on lowlevel aspects of the computing system in terms of effectively utilizing specific hardware capabilities and the development of operating systems and compilers to reduce energy usage consistent with performance constraints. Only a modest amount of work to date 
has addressed energy and power consumption in parallel and distributed simulations. Some work has focused on characterizing power consumption for scientific computing applications [Feng et al. 2005; Dongarra et al. 2012; Esmaeilzadeh et al. 2012]. Early work examined power consumption for disseminating state information in distributed virtual environments, highlighting dead-reckoning algorithms and tradeoffs between state consistency and power consumption [Shi et al. 2003]. More recent work examined power consumption related to the implementation of data distributed management (DDM) services defined on the High-Level Architecture [Neal et al. 2014] and time management [Fujimoto and Biswas 2015].

Power-aware parallel and distributed simulation for embedded and highperformance computing applications represents a new direction of research with many open questions. As noted earlier, DDDAS deployments may utilize simulations embedded in the physical environment, or use of mobile simulations for state prediction. The design of a simulation (e.g., in terms of data structures and algorithms with respect to energy and power consumption) has not been extensively studied. There are a variety of modeling approaches that may be utilized to model the physical system. For example, for predictive transportation simulations, the network may be modeled using macroscopic, mesoscopic, or microscopic simulation models. These approaches represent tradeoffs among modeling detail, computational performance, and energy use. The relationships among model detail and fidelity and power consumption are not well understood. These tradeoffs must consider analysis of data produced by the simulation, which will likely be greater for more detailed models, in addition to the execution of the simulation model. Further, modeling results must be produced in a timely fashion when the model is used for managing operational systems, imposing real-time constraints on model execution time.

Online simulations incorporate live data streams into the simulation to characterize the current system and/or to calibrate and adapt the simulation to improve model predictions. The frequency and amount of data utilized by the simulation will have a large impact on power consumption and energy use. In addition to the power required to transmit the data, data analysis algorithms will also consume energy to process incoming data. These factors suggest a tradeoff between model accuracy and energy use that varies according to the frequency and amount of data utilized by the simulation. Further, these tradeoffs among model fidelity, execution time, and power consumption may change dynamically over time. For instance, in transportation applications, congested traffic conditions may result in increased volumes of data, especially if crowdsourced information is used, and may require more detailed, sophisticated models to accurately assess and evaluate traffic conditions.

As discussed earlier, parallel discrete event simulations and distributed simulations utilizing time management services require a synchronization algorithm to ensure correctness. These synchronization algorithms require additional interprocessor communication that can significantly affect the amount of energy that is consumed. For conservative algorithms, this communication may come in the form of null messages or barrier synchronizations and reduction computations to determine when it is safe to process events. Prior work in parallel discrete event simulation has focused on speedup of the simulation relative to a sequential execution. The power consumed by different conservative synchronization algorithms is not well understood, and little consideration has been made to date concerning the design of synchronization algorithms taking into consideration power consumption and energy use [Fujimoto and Biswas 2015]. Similarly, little is known concerning power consumption of optimistic synchronization algorithms. While optimistic synchronization algorithms do not require null messages and global synchronizations occur less frequently in the form of global virtual time computations, algorithms such as Time Warp will require computations that are later 
rolled back and the transmission and processing of antimessages that can have a significant impact on power consumption and energy use that are not present in conservative algorithms. These factors raise new questions concerning the suitability of optimistic and conservative synchronization with respect to energy and power consumption, and raise the question of how best to design synchronization algorithms that minimize energy consumption.

\subsection{Creating Rapidly Composable Distributed Simulations}

Challenge 6. Enable rapid composition of separately developed simulation models for execution in parallel or distributed simulation environments.

A long-standing problem in distributed simulation concerns the desire to integrate different simulators into a single simulation environment. One of the initial, motivating applications for this capability arose in the defense community, especially for training purposes. Tank simulators, flight simulators, computer-generated forces, and a variety of other models may be used to create a distributed virtual environment into which personnel are embedded to train for hypothetical scenarios and situations. To this day, the desire for seamless integration of live, virtual, and constructive (LVC) simulations remains an important objective in distributed simulation for defense.

Another application of great importance today concerns the development of more resilient and sustainable cities. Cities are undergoing a revolution with new developments such as the increased deployment of electric and autonomous vehicles, widespread instrumentation providing detailed information concerning energy usage, growing use of renewable energy sources relative to consumption of fossil fuels, and new modes of interaction and information propagation via social networks, to name a few. It is well understood that it is not sufficient to study individual infrastructures (e.g., transportation, water, energy, telecommunications, food production) or social processes (e.g., economics, social attitudes concerning the adoption of more sustainable practices, urban growth, land use) of modern cities in isolation because there are important interdependencies that must be considered. Energy production relies heavily on water for cooling fossil-fueled and nuclear power plants as well as for hydro-electric power generation. In many cases, these uses compete for the same water as the agriculture and food production industries, with impacts that affect the cost of living for consumers and their ability to purchase other technologies that can lead to more sustainable growth and development. While the complex systems arising within each sector are understood to varying degrees, systems of complex systems are very poorly understood, and it is clear that modeling and simulation will play a critical role in studying their behavior. The importance of interactions among infrastructures has similarly been recognized in creating cities that are more resilient to natural and human-caused disasters and emergencies, and integration of infrastructure simulations remains an important challenge today [Adam et al. 2013].

Rapidly creating credible simulation models by composing existing simulation models continues to be challenging. Ideally, one would like a system that could rapidly transform high-level specifications of the system under investigation into efficient parallel/distributed simulation code where aspects such as model selection and configuration, composition, and code generation are automated. This raises a number of issues. A comprehensive discussion of this topic is well beyond the scope of this article. Here, we briefly highlight work in three key areas: (1) defining what one means by composition, (2) issues concerning the integration of different types of models, and (3) fundamental limits of composition and automated model generation.

In Petty and Weisel [2003], composability is defined as "the capability to select and assemble simulation components in various combinations into valid simulation systems to satisfy specific user requirements." Composability is not a binary, yes/no prop- 


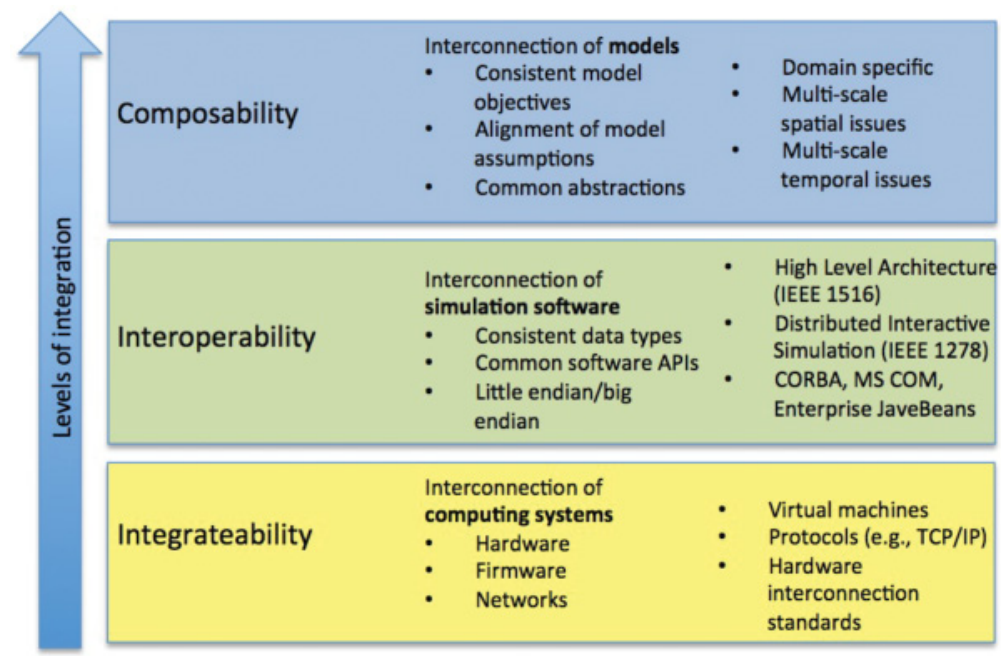

Fig. 3. Levels of integration in distributed simulations.

erty, but rather is characterized by degrees. Three levels of integration are depicted in Figure 3 based on the hierarchy proposed in Page et al. [2004]. These levels are elaborated upon in Tolk [2012] to define seven levels that highlight several key issues to achieving composability. In this figure, the first level, termed integratability, focuses on the interconnection of computing systems at the hardware, firmware, and networking levels; standardized hardware interfaces and network protocols enable communication among computer systems. This level includes standards such as hardware cable and physical connections as well as networking protocols such as TCP/IP. The second level, interoperability, addresses software interoperability issues by defining common data standards and data types to enable simulations to communicate, exchange data, and synchronize with each other. It includes standards such as DIS and HLA, for example. Syntactic interoperability refers to the ability to exchange common symbols among systems. Semantic interoperability refers to the ability to interpret information exchanged among systems, and requires definitions of common terms and the translation of symbols exchanged among systems to these terms.

The third level, composability, is concerned with the composition of separately developed models of subsystems to create system models. A long-standing goal of the distributed simulation community has been to achieve "plug-and-play" interoperability where one is able to automatically compose separately developed simulations independent of the context into which they are embedded. This implies model composability and is far from the capabilities of current technologies without substantial time and effort, as elaborated upon next.

Work in multimodeling or multiparadigm modeling provides some insight into the problems of constructing distributed simulations where the components of the system are modeled using different modeling approaches. One example is hybrid systems composed of continuous models based on differential equations to model (say) the dynamics of a mechanical system, and discrete events are used to model abrupt changes such as thresholds being exceeded or users invoking commands through a control panel. Multimodeling has been widely used in embedded system design and cyber-physical systems where models, computations, and networks are combined with physical devices. Computer-Automated Multi-Paradigm Modeling (CAMPaM) strives to create domain-independent frameworks to support the development of heterogeneous models 
[Mosterman and Vangheluwe 2004]. CAMPaM systems must address three issues that arise in such systems:

- Multiple modeling formalisms. At the heart of multimodeling is combining models using formalisms to create hybrid models, for example, combining discrete event and differential equation-based continuous time models as discussed earlier. Many modeling formalisms have been developed, for example, state charts, Petri nets, finite state machines, cellular automata, and bond graphs, to mention a few. Different system components may be represented using different modeling formalisms, necessitating the ability to compose these formalisms.

- Multiple levels of abstraction. Separate from the choice of modeling formalism is the level of abstraction used to model the system. Different levels of abstraction may be defined in time and space. The relationship among these levels of abstraction must be understood and mechanisms to translate among them at the interface between the models must be developed.

- Metamodels and translators. A model is an instance of a modeling language that describes a system, and is typically based on a specific modeling formalism. A metamodel defines the modeling language. The same metamodeling language can describe multiple modeling languages, and thus can be used to specify systems that utilize multiple formalisms [Vangheluwe and Lara 2002; Cetinkaya and Verbraeck 2011]. The metamodel provides a means of describing the overall system composed of the different constituent modeling formalisms supported by the modeling system. Compilers or translators provide a means to automatically transform a metamodel for a system into a simulation model utilizing multiple formalisms.

Ptolemy II is a well-known example of a multimodeling framework that supports integrated treatment of several modeling formalisms such as finite state machines, discrete event models, and differential equation-based models, among others [Ptolemaeus 2014]. AToM3 is a second example of metamodels used to support multiformalism modeling and simulation [De Lara and Vangheluwe 2002].

Multimodeling frameworks address issues of heterogeneity but typically assume new models are developed entirely within that framework. Standards such as HLA and DIS focus on combining separately developed simulations but fall well short of model composition. Extensive manual effort is required to create and validate the resulting distributed simulation. Approaches that combine these approaches and provide automated code generation to compose separately developed models may provide a path to simplify or automate composition while providing some assurances concerning the result. Composition of arbitrary models is difficult if not impossible, suggesting that constraints on the development of the original models may be required to enable subsequent composition. Further research is required to explore this issue.

On a cautionary note, there are limits as to how far one can go with automated model composition. Some researchers have examined the underlying theory in order to characterize the difficulty and limitations of techniques to automate the creation of simulation models in general, and composed models in particular. A formalism called the condition specification (CS) is described in Overstreet and Nance [1985] that provides a vehicle to address issues concerning the complexity of simulations. CS has an equivalent Turing Machine specification. It has been used to show that several problems are undecidable; for example, no procedures can be developed to show a model specification is finite (will terminate), ambiguous (two nonequivalent implementations are possible), complete (no execution can reach a state not described by the specification), or connected (the specification does not consist of several independent systems) [Overstreet 1982]. Page and Opper [1999] show that for one class of composed simulations, determining if a set of components meets the objectives stated in a system specification is NP-complete. 
There has been much progress in the development of techniques and tools to facilitate integration of simulations at the two lower levels of the hierarchy shown in Figure 3. However, future research will likely need to focus on federating simulations at the conceptual model level to achieve model composition. This work must necessarily involve increased consideration of the domain of application. Application-specific standards will need to be developed to augment other simulation interoperability standards that are already in place.

\section{CONCLUDING REMARKS}

The parallel and distributed simulation field has evolved over the last several decades, and many impressive accomplishments have been achieved. The field has matured. However, the technology has yet to achieve its fullest potential. The world has changed. From below in the software stack, new computing platforms such as massively parallel machines, GPUs, cloud computing, and emergence of the "third platform" are making transformative changes to computing as a whole. From above, new applications such as large-scale simulation of scale-free networks and modeling systems of systems arising in smart cities impose new needs for this technology as well as new requirements and constraints. These changes have created new research challenges requiring new lines of inquiry and discovery. With this article, we have reviewed a few key contributions to the field in the past and attempt to articulate research challenges for the future.

Moreover, we believe there is value in identifying and building consensus around key research challenges for the community to address. This article may help to serve as a first step toward establishing such a common research agenda for the parallel and distributed simulation field.

\section{ACKNOWLEDGMENTS}

The author gratefully acknowledges support from the National Science Foundation (most recently awards 1258425, 1441208, and 1462503) and the Air Force Office of Scientific Research (FA9550-13-1-0100) and comments by the anonymous referees and editors that led to improvements in this article.

\section{REFERENCES}

N. Adam, R. Stiles, A. Zimdars, R. Timmons, J. Leung, G. Stachnick, J. Merrick, R. Coop, V. Slavin, T. Kruglikov, J. Galmiche, and S. Mehrotra. 2013. Consequence analysis of complex events on critical U.S. Infrastructure. Communications of the ACM, 56, 6, 83-91.

G. Allen. 2007. Building a dynamic data driven application system for hurricane forecasting. In Computational Science (ICCS'07). Y. Shi, G. D. V. Albada, J. Dongarra, and P. M. A. Sloot (Eds.). Springer, Berlin, 1034-1041.

H. Aydt, S. J. Turner, W. Cai, and M. Y. H. Low. 2009. Research issues in symbiotic simulation. Winter Simulation Conference, 1213-1222.

A. L. Barabasi and R. Albert. 1999. Emergence of scaling in random networks. Science 286, 5439, 509-512.

P. D. Barnes, C. D. Carothers, D. R. Jefferson, and J. M. LaPre. 2013. Warp speed: Executing time warp on 1,966,080 cores. Principles of Advanced Discrete Simulation, 327-336.

K. Bhatti, C. Belleudy, and M. Auguin. 2010. Power management in real time embedded systems through online and adaptive interplay of DPM and DVFS policies. International Conference on Embedded and Ubiquitous Computing, 184-191.

K. R. Bisset, J. Chen, X. Feng, V. S. A. Kumar, and M. V. Marathe. 2009. Epifast: A fast algorithm for large scale realistic epidemic sim- ulations on distributed memory systems. International Conference of Supercomputing, 430-439.

A. Boukerche and R. E. De Grande. 2009. Dynamic load balancing using grid services for hla-based simulations on large-scale distributed systems. International Symposium on Distributed Simulation and Real Time Applications, 175-183.

C. Brun, T. Artés, T. Margalef, and A. Cortés. 2012. Coupling wind dynamics into a DDDAS forest fire propagation prediction system. In Proceedings of the International Conference on Compuational Science.

R. E. Bryant. 1977. Simulation of packet communications architecture computer systems. MIT-LCS-TR-188. 
C. D. Carothers and R. M. Fujimoto. 2000. Efficient execution of time warp programs on heterogeneous, now platforms. IEEE Transactions on Parallel and Distributed Systems 11, 3, 299-317.

D. Cetinkaya and A. Verbraeck. 2011. Metamodeling and model transformations in modeling and simulation. Winter Simulation Conference, 3048-3058.

K. M. Chandy and J. Misra. 1978. Distributed simulation: A case study in design and verification of distributed programs. IEEE Transactions on Software Engineering SE-5, 5, 440-452.

A. Chaturvedi, A. Mellema, S. Filatyev, and J. Gore. 2006. DDDAS for fire and agent evacuation modeling of the rhode island nightclub fire. In Computational Science (ICCS'06). V. N. Alexandrov, G. D. V. Albada, P. M. A. Sloot, and J. Dongarra (Eds.). Springer, Berlin, 433-439.

K.-M. Cho, C.-H. Liang, J.-Y. Huang, and C.-S. Yang. 2011. Design and implementation of a general purpose power-saving scheduling algorithm for embedded systems. IEEE International Conference on Signal Processing, Communications and Computing, 1-5.

M. Chung and Y. Chung. 1991. An experimental analysis of simulation clock advancement in parallel logic simulation on an simd machine. Advances in Parallel and Distributed Simulation, SCS Simulation Series. 23, 125-132.

R. Cohen and S. Havlin. 2003. Scale-free networks are ultrasmall. Physics Review Letters 90, 5, 058701.

J. Cortial, C. Farhat, L. J. Guibas, and M. Rajashekhar. 2007. Compressed sensing and time-parallel reducedorder modeling for structural health monitoring using a DDDAS. In Computational Science (ICCS'07). Y. Shi, G. D. V. Albada, J. Dongarra, and P. M. A. Sloot (Eds.). Springer, Berlin, 1171-1179.

M. Curtis-Maury, A. Shah, F. Blagojevic, D. S. Nikolopoulos, B. R. de Supinski, and M. Schulz. 2008. Prediction models for multi-dimensional power-performance optimization on many cores. Proceedings of the 17th International Conference on Parallel Architectures and Compilation Techniques. ACM, New York, NY, 250-259.

K. Czechowski and R. Vuduc. 2013. A theoretical framework for algorithm-architecture co-design. 2013 IEEE 27th International Symposium on Parallel Distributed Processing (IPDPS'13), 791-802.

D. W. Bauer Jr., C. D. Carothers, and A. Holder. 2009. Scalable time warp on blue gene supercomputers. Principles of Advanced and Distributed Simulation, 35-44.

G. D'Angelo. 2011. Parallel and distributed simulation from many cores to the public cloud. International Conference on High Performance Computing and Simulation, 14-23.

G. D'Angelo and S. Ferretti. 2009. Simulation of scale-free networks. In Proceedings of the 2nd Internatinoal Conference on Simulation Tools and Techniques. ICST, Rome, Italy.

F. Darema. 2004. Dynamic data driven applications systems: A new paradigm for application simulations and measurements. International Conference on Computational Science.

J. De Lara and H. Vangheluwe. 2002. AToM3: A tool for multi-formalism and meta-modelling. In Proceedings of the Fundamental Approaches to Software Engineering. R.-D. Kutsche and H. Weber (Eds.). Springer. 2306, 174-188.

R. Dobrescu, S. Taralunga, and S. Mocanu. 2008. Parallel internet traffic simulator with self-similar scalefree network models. WSEAS Transactions on Advances in Engineering Education 5, 2, 61-68.

J. Dongarra, H. Ltaief, P. Luszczek, and V. M. Weaver. 2012. Energy footprint of advanced dense numerical linear algebra using tile algorithms on multicore architecture. In The 2nd International Conference on Cloud and Green Computing.

C. C. Douglas, R. A. Lodder, J. D. Beezley, J. Mandel, R. E. Ewing, Y. Efendiev, G. Qin, M. Iskandara-ni, J. Coen, A. Vodacek, M. Kritz, and G. Haase. 2006. DDDAS approaches to wildland fire modeling and contaminant tracking. In Proceedings of the 2006 Winter Simulation Conference.

M. Erazo, R. Rong, and J. Liu. 2015 Symbiotic network simulation and emulation. ACM Transactions on Modeling and Computer Simulation 26, 1.

J. Ekanayake and G. Fox. 2009. High performance parallel computing with clouds and cloud technologies. Department of Computer Science, Indiana University.

H. Esmaeilzadeh, T. Cao, X. Yang, S. M. Blackburn, and K. S. McKinley. 2012. Looking back and looking forward: Power, performance, and upheaval. Communications of the ACM 55, 7, 105-114.

M. Falk, M. Ott, T. Ertl, M. Klann, and H. Koeppl. 2011. Parallelized agent-based simulation on CPU and graphics hardware for spatial and stochastic models in biology. In 9th International Conference on Computational Methods in Systems Biology, 73-82.

M. Faloutsos, P. Faloutsos, and C. Faloutsos. 1999. On power-law relationships of the internet topology. SIGCOMM Computer Communications Review 29, 4, 251-262.

S. Feng, Y. Di, Yuanchang, and Z. X. Meng. 2010. Remodeling traditional RTI software to be with paas architecture. In International Conference on Computer Science and Information Technology, $511-515$. 
X. Feng, R. Ge, and K. W. Cameron. 2005. Power and energy profiling of scientific applications on distributed systems. In Proceedings of the 19th IEEE International Parallel and Distributed Processing Symposium (IPDPS'05), 34.

R. M. Fujimoto, M. Hunter, J. Sirichoke, M. Palekar, H.-K. Kim, and W. Suh. 2007. Ad hoc distributed simulations. Principles of Advanced and Distributed Simulation.

R. M. Fujimoto. 1990. Performance of time warp under synthetic workloads. In Proceedings of the SCS Multiconference on Distributed Simulation.

R. M. Fujimoto. 1993. Parallel discrete event simulation: Will the field survive? ORSA Journal on Computing $5,3,213-230$.

R. M. Fujimoto. 2000. Parallel and Distributed Simulation Systems. Wiley Interscience.

R. M. Fujimoto. 2015. Parallel and distributed simulation. In Winter Simulation Conference, 45-59.

R. M. Fujimoto and A. Biswas. 2015. An empirical study of energy consumption in distributed simulations. In International Symposium on Distributed Simulation and Real-Time Applications.

R. M. Fujimoto, D. Lunceford, E. Page, and A. Uhrmacher (Eds.). 2002. Grand Challenges in Modeling and Simulation. Technical Report 350, Schloss Dagstuhl, Seminar No. 02351.

R. M. Fujimoto, A. W. Malik, and A. J. Park. 2010. Parallel and distributed simulation in the cloud. SCS Modeling and Simulation Magazine, International Society for Modeling and Simulation, 1, 3.

R. M. Fujimoto, K. Perumalla, A. Park, H. Wu, M. H. Ammar, and G. F. Riley. 2003. Large-scale network simulation: How big? how fast? In Symposium on Modeling, Analysis, and Simulation of Computer and Telecommunication Systems.

K. Fürlinger, C. Klausecker, and D. Kranzlmüller. 2011. Towards energy efficient parallel computing on consumer electronic devices. In Proceedings of the 1st International Conference on Information and Communication on Technology for the Fight Against Global Warming. Springer-Verlag, Berlin, 1-9.

I. Grasso, P. Radojkovic, N. Rajovic, I. Gelado, and A. Ramirez. 2014. Energy efficient HPC on embedded socs: Optimization techniques for mali GPU. In 2014 IEEE 28th International Parallel and Distributed Processing Symposium, 123-132.

H. Guclu, G. Korniss, Z. Toroczkai, and M. A. Novotny. 2004. Small-world synchronized computing networks for scalable parallel discrete-event simulations. In Complex Networks, E. Ben-Naim, H. Frauenfelder, and Z. Toroczkai (Eds.). Springer-Verlag, Berlin. 650, 255-275.

K. A. Hawick, A. Leist, and D. P. Playne. 2011. Regular lattice and small- world spin model simulations using CUDA and GPUs. International Journal of Parallel Programming 39, 2, 183-201.

H. He, R. Li, X. Dong, Z. Zhang, and H. Han. 2012. An efficient and secure cloud-based distributed simulation system. Journal of Applied Mathematics \& Information Sciences 6, 3, 729-736.

M. D. Hill and M. R. Marty. 2008. Amdahl's law in the multicore era. Computer 41, 7, 33-38.

T. Hruz, S. Geisseler, and M. Schöngens. 2010. Parallelism in simulation and modeling of scale-free complex networks. Parallel Computing 36, 8, 469-485.

S. Hua and G. Qu. 2003. Approaching the maximum energy saving on embedded systems with multiple voltages. In IEEE/ACM International Conference on Computer-Aided Design, 26.

Y.-L. Huang, M. Hunter, C. Alexopoulos, and R. M. Fujimoto. 2010. Ad hoc distributed simulation of queueing networks. Principles of Advanced and Distributed Simulation.

IEEE Std. 1278.1-1995. 1995. IEEE Standard for Distributed Interactive Simulation-Application Protocols. Institute of Electrical and Electronics Engineers, New York, NY.

IEEE Std. 1278.2-1995. 1995. IEEE Standard for Distributed Interactive Simulation - Communication Services and Profiles. Institute of Electrical and Electronics Engineers, New York, NY.

IEEE Std. 1516-2010. 2010. IEEE Standard for Modeling and Simulation (M\&S) High Level Architecture (HLA) - Framework and Rules. Institute of Electrical and Electronics Engineers, New York, NY.

IEEE Std. 1516.1-2010. 2010. IEEE Standard for Modeling and Simulation (M\&S) High Level Architecture (HLA) - Interface Specification. Institute of Electrical and Electronics Engineers, New York, NY.

IEEE Std. 1516.2-2010. 2010. IEEE Standard for Modeling and Simulation (M\&S) High Level Architecture (HLA) - Object Model Template (OMT) Specification. Institute of Electrical and Electronics Engineers, New York, NY.

V. Janapa Reddi, B. C. Lee, T. Chilimbi, and K. Vaid. 2010. Web search using mobile cores: Quantifying and mitigating the price of efficiency. SIGARCH Computer Architecture News 38, 3, 314-325.

D. Jefferson. 1985. Virtual time. ACM Transactions on Programming Languages and Systems 7, 3, 404-425.

H. Jia-Wei and H. T. Kung. 1981. I/O complexity: The red-blue pebble game. In Proceedings of the 13th Annual ACM symposium on Theory of Computing (STOC'81). ACM Press, New York, NY, 326-333. 
D. Jin and D. Nicol. 2015. Parallel simulation and virtual-machine-based emulation of software-defined networks. ACM Transactions on Modeling and Computer Simulation 26, 1, Article 8.

D. W. Jones. 1986. An empirical comparison of priority-queue and event-set implementations. Communications of the ACM 29, 4, 300-311.

I.-Y. Jung, B.-J. Han, and C.-S. Jeong. 2014. Provisioning on-demand HLA/RTI simulation environment on cloud for distributed-parallel computer simulations. Mobile, Ubiquitous, and Intelligent Computing, 329-334.

F. Kamrani and R. Ayani. 2007. Using on-line simulation for adaptive path planning of UAVs. In Proceedings of the 11th IEEE International Symposium on Distributed Simulation and Real-Time Applications.

H. Kitano. 2002. Systems biology: A brief overview. Science 295, 5560, 1662-1664.

G. Kunz, D. Schemmel, J. Gross, and K. Wehrle. 2012. Multi-level parallelism for time and cost efficient parallel discrete event simulation on GPUs. Principles of Advanced and Distributed Simulation, $23-32$.

G. Kunz, M. Stoffers, O. Landsiedel, K. Wehrle, and J. Gross. 2016. Parallel expanded event simulation of tightly coupled systems. ACM Transaction on Modeling and Computer Simulation 26, 2, Article 12.

P. Lendermann, M. Y. H. Low, B. P. Gan, N. Julka, L.-P. Chan, L. H. Lee, S. J. E. Taylor, S. J. Turner, W. Cai, X. Wang, T. Hung, L. F. McGinnis, and S. Buckley. 2005. An integrated and adaptive decisionsupport framework for high-tech manufacturing and service networks. In Proceedings of the 2005 Winter Simulation Conference.

B.-H. Li, X. Chai, B. Hou, C. Yang, T. Li, T. Lin, Z. Zhang, Y. Zhang, W. Zhu, and Z. Zhao. 2013. Research and application on cloud simulation. Summer Computer Simulation Conference, 157-170.

L. Li, D. Alderson, J. Doyle, and W. Willinger. 2004. A first-principles approach to understanding the internet's router-level topology. ACM Computer Communication Review 34, 3-14.

X. Li, W. Cai, and S. Turner. 2013. GPU acceleratedthree-stage execution model for event-parallel simulation. Principles of Advanced Discrete Simulation, 57-66.

J. Liu, Y. Liu, Z. Du, and T. Li. 2014. GPU-assisted hybrid network traffic model. Principles of Advanced Discrete Simulation, 63-74.

J. Liu and R. Rong. 2012. Hierarchical composite synchronization. Principles of Advanced and Distributed Simulation, 3-12.

X. Liu and A. A. Chien. 2004. Realistic large-scale online network simulation. In Proceedings of the 2004 ACM/IEEE Conference on Supercomputing.

X. Liu, X. Qiu, B. Chen, and K. Huang. 2012. Cloud-based simulation: The state-of-the-art computer simulation paradigm. Principles of Advanced and Distributed Simulation, 71-74.

C. Lively, V. Taylor, X. Wu, H.-C. Chang, C.-Y. Su, K. Cameron, S. Moore, and D. Terpstra. 2014. E-AMOM: An energy-aware modeling and optimization methodology for scientific applications. Computer Science Research and Development 29, 3-4, 197-210.

M. Y. H. Low, K. W. Lye, P. Lendermann, S. J. Turner, R. T. W. Chim, and S. H. Leo. 2005. An agentbased approach for managing symbiotic simulation of semiconductor assembly and test operation. In Proceedings of the 14th International Joint Conference on Autonomous Agents and Multiagent Systems. Association for Computing Machinery, New York, 85-92.

B. D. Lubachevsky. 1989. Efficient distributed event-driven simulations of multiple-loop networks. Communications of the ACM 32, 1, 111-123.

G. R. Madey, M. B. Blake, C. Poellabauer, H. Lu, R. R. McCune, and Y. Wei. 2012. Applying DDDAS principles to command, control and mission planning for UAV swarms. In Proceedings of the International Conference on Compuational Science.

A. W. Malik, A. J. Park, and R. M. Fujimoto. 2010. An optimistic parallel simulation protocol for cloud computing environments. SCS Modeling and Simulation Magazine, International Society for Modeling and Simulation, $1,4$.

J. Mandel, J. D. Beezley, A. K. Kochanski, V. Y. Kondratenko, and M. Kim. 2012. Assimilation of perimeter data and coupling with fuel moisture in a wildland fire - atmosphere DDDAS. In Proceedings of the International Conference on Compuational Science.

D. C. Miller and J. A. Thorpe. 1995. SIMNET: The advent of simulator networking. In Proceedings of the IEEE 83, 8, 1114-1123.

K. L. Morse and M. Zyda. 2001. Multicast grouping for data distribution management. SIMPRA - Journal of Simulation Practice and Theory, Fall.

P. Mosterman and H. Vangheluwe. 2004. Computer automated multi-paradigm modeling: An introduction. Simulation: Transactions of the Society for Modeling and Simulation International 80, 9, 433-450.

S. Neal, G. Kanitkar, and R. M. Fujimoto. 2014. Power consumption of data distribution management for on-line simulations. Principles of Advanced Discrete Simulation, 197-204. 
D. M. Nicol and P. Heidelberger. 1996. Parallel execution for sequential simulators. ACM Transactions on Modeling and Computer Simulation 6, 3, 210-242.

D. M. Nicol, C. Micheal, and P. Inouye. 1989. Efficient aggregaton of multiple LPs in distributed memory parallel simulations. Winter Simulation Conference, 680-685.

L. Niu and G. Quan. 2004. Reducing both dynamic and leakage energy consumption for hard real-time systems. International Conference on Compilers, Architecture, and Synthesis for Embedded Systems, 140-148.

NVIDIA. 2014. Whitepaper: NVIDIA GeForce GTX 750 Ti.

C. M. Overstreet. 1982. Model Specification and Analysis for Discrete Event Simulation, Ph.D. dissertation, Virginia Tech, Blacksburg.

C. M. Overstreet and R. E. Nance. 1985. A specification language to assist in analysis of discrete event simulation models. Communications of the ACM 28, 2, 190-201.

E. H. Page, R. Briggs, and J. A. Tufarolo. 2004. Toward a family of maturity models for the simulation interconnection problem. Spring 2004 Simulation Interoperability Workshop, IEEE CS Press.

E. H. Page and J. M. Opper. 1999. Observations on the complexity of composable simulation. Winter Simulation Conference, 553-560.

A. Park and R. M. Fujimoto. 2012. Efficient master/worker parallel discrete event simulation on metacomputing systems. IEEE Transactions on Parallel and Distributed Systems 23, 5.

H. Park and P. Fishwick. 2011. An analysis of queuing network simulation using GPU-based hardware acceleration. ACM Transactions on Modeling and Computer Simulation 21, 3.

A. Pellegrini, R. Vitali, S. Peluso, and F. Quaglia. 2012. Transparent and efficient shared-state management for optimistic simulations on multi-core machines. IEEE Symposium on Modeling, Analysis \& Simulation of Computer and Telecommunication Systems, 134-141.

K. S. Perumalla. 2007. Scaling time warp-based discrete event execution to $10^{* *} 4$ processors on a blue gene supercomputer. In Proceedings of the ACM Computing Frontiers Conference. Ischia, Italy.

K. S. Perumalla, G. A. Brandon, B. Y. Srikanth, and K. S. Sudip. 2009. GPU-based real-time execution of vehicular mobility models in large-scale road network scenarios. Principles of Advanced and Distributed Simulation, 95-103.

K. S. Perumalla and S. K. Seal. 2010. Reversible parallel discrete-event execution of large-scale epidemic outbreak models. Principles of Advanced and Distributed Simulation, 106-113.

K. S. Perumalla, A. J. Park, and V. Tipparaju. 2014. Discrete event execution with one-sided and two-sided GVT Algorithms on 216,000 processor cores. ACM Transactions on Modeling and Computer Simulation, $24,3,16: 1-16: 25$.

M. D. Petty and E. W. Weisel. 2003. A composability lexicon. IEEE Spring Simulation Interoperability Workshop.

R. Pienta and R. M. Fujimoto. 2013. On the parallel simulation of scale-free networks. Principles of Advanced and Discrete Simulation.

B. Plale, D. Gannon, and D. Reed. 2005. Towards dynamically adaptive weather analysis and forecasting in LEAD. International Conference on Computational Science.

C. Ptolemaeus (Ed.). 2014. System Design, Modeling, and Simulation using Ptolemy II. Ptolemy.Org.

G. Quan and X. Hu. 2001. Energy efficient fixed- priority scheduling for real-time systems on variable voltage processors. Design Automation Conference, 828-833.

G. Riley, M. Ammar, R. M. Fujimoto, A. Park, K. Perumalla, and D. Xu. 2004. A federated approach to distributed network simulation. ACM Transactions on Modeling and Computer Simulation 14, 1, 116148.

B. B. Romdhanne, M. S. M. Bouksiaa, N. Nikaein, and C. Bonnet. 2013. Hybrid scheduling for event-driven simulation over heterogeneous computers. Principles of Advanced Discrete Simulation, 47-56.

A. Santoro and F. Quaglia. 2012. Transparent optimistic synchronization in the high-level architecture via time-management conversion. ACM Transaction on Modeling and Simulation 22, 4, 21:21-21:26.

M. Satyanarayanan, P. Bahl, R. Caceres, and N. Davies. 2009. The case for VM-based cloudlets in mobile computing. IEEE Pervasive Computing 8, 14-23.

Scalable Network Technologies. 2012. QualNet. 2012. http://www.scalable-networks.com/content/products/ qualnet.

W. Shi, K. S. Perumalla, and R. M. Fujimoto. 2003. Power-Aware State Dissemination in Mobile Distributed Virtual Environments. Workshop on Parallel and Distributed Simulation, San Diego.

G. Siganos, M. Faloutsos, P. Faloutsos, and C. Faloutsos. 2003. Power laws and the AS-Level internet topology. IEEE/ACM Transactions on Networking 11, 4, 514-524. 
K. Soramaki, M. L. Bech, J. Arnold, R. J. Glass, and W. E. Beyeler. 2007. The topology of interbank payment flows. Physica A: Statistical Mechanics and Its Applications 379, 1, 317-333.

L. Stanisic, B. Videau, J. Cronsioe, A. Degomme, V. Marangozova-Martin, A. Legrand, and J.-F. Mehaut. 2013. Performance analysis of HPC applications on low-power embedded platforms. In Design, Automation Test in Europe Conference Exhibition (DATE'13), 475-480.

W. Suh, M. Hunter, and R. M. Fujimoto. 2014. Ad hoc distributed simulation for transportation system monitoring and near-term prediction. Simulation Modeling Practice and Theory 41, 1-14.

A. Tolk (Ed.). 2012. Engineering Principles of Combat Modeling and Distributed Simulation. John Wiley and Sons, Hoboken, NJ.

A. Tolk. 2012. Challenges of distributed simulation. Engineering Principles of Combat Modeling and Distributed Simulation. John Wiley and Sons.

O. S. Unsal. 2008. System-Level Power-Aware Computing In Complex Real-Time and Multimedia Systems. Doctor of Philosophy Doctoral Dissertation, University of Massachusetts.

H. Vangheluwe and J. D. Lara. 2002. Meta-models are models too. Winter Simulation Conference, 597-605.

K. Vanmechelen, S. De Munck, and J. Broeckhove. 2012. Conservative distributed discrete event simulation on amazon EC2. In International Symposium on Cluster, Cloud, and Grid Computing, 853-860.

G. Vulov, C. Hou, R. Vuduc, D. Quinlan, R. M. Fujimoto, and D. Jefferson. 2011. The backstroke framework for source level reverse computation applied to parallel discrete event simulation. In Winter Simulation Conference.

E. Walker. 2008. Benchmarking amazon EC2 for high performance scientific computing. http://www. usenix.org/publications/login/2008-10/openpdfs/walker.pdf.

X. F. Wang and G. Chen. 2003. Complex networks: Small-world, scale-free and beyond. IEEE Circuits and Systems Magazine 3, 1, 6-20.

Y. Xu, G. Tan, X. Li, and X. Song. 2014. Mesoscopic traffic simulation on CPU/GPU. Principles of Advanced Discrete Simulation, 39-49.

V. Yau. 1999. Automating parallel simulation using parallel time streams. ACM Transactions on Modeling and Computer Simulation, 9, 2, 171-201.

T. Ye, H. Kaur, S. Kalyanaraman, and M. Yuksel. 2008. Large-scale network parameter configuration using an on-line simulation framework. IEEE/ACM Transactions on Networking 16, 777-790.

S. B. Yoginath and K. S. Perumalla. 2013. Empirical evaluation of conservative and optimistic discrete event execution on cloud and VM platforms. Principles of Advanced Discrete Simulation, 201-210.

A. Yoo and K. W. Henderson. 2010. Parallel generation of massive scale-free graphs. CoRR.

L. Zhang, X. Deng, J. Yu, and X. Wu. 2011. The degree and connectivity of internet's scale-free topology. Chinese Physics B 20, 4, 048902.

P. Zou, Y.-S. Lu, L.-D. Wu, L.-l. Chen, and Y.-P. Yao. 2013. Epidemic simulation of a large-scale social contact network on gpu clusters. Simulation: Transactions of the Society for Modeling and Simulation International 89, 10, 1154-1172.

Received April 2015; revised October 2015; accepted December 2015 